\title{
Influencia de las Redes Sociales en la Rentabilidad de las Empresas, caso: Empresas ecuatorianas de Retail
}

\section{Social Networks Influence in Business Profitability, case: Ecuadorian Retail Companies}

\author{
Joselyn Ximena Valarezo Romero \\ Erick Daniel Cedeño Rivera \\ Ernesto Felipe Novillo Maldonado \\ https://orcid.org/0000-0002-7778-729X \\ Universidad Técnica de Machala, Ecuador
}

Autor por correspondencia: jxvalarezo_est@utmachala.edu.ec; edcedeno_est@utmachala.edu.ec; enovillo@utmachala.edu.ec Fecha de recepción: 12 de febrero de 2020 - Fecha de aceptación: 29 de abril de 2020

\section{Resumen}

El presente trabajo tiene por objetivo analizar el nivel de influencia de las redes sociales ante el éxito empresarial, ha sido desarrollado a través de dos herramientas de investigación, la primera consiste en un cuadro de observación hacia las redes sociales gestionadas por las empresas seleccionadas como objeto de estudio, cuyo análisis se realizó en base a cuatro ítems: actividad, comunidad, visibilidad e interactividad; en cuanto a la segunda herramienta, se destaca el uso de una encuesta de escala Likert, que ha permitido conocer la percepción de los consumidores referente al uso de redes sociales como herramienta de información para sus tendencias de compra. Los instrumentos aplicados, permitieron determinar que la gestión en redes sociales no representa un punto clave frente al éxito empresarial, no obstante, de las 5 empresas analizadas, menos del 50\% gestiona redes sociales, permitiendo rechazar la hipótesis planteada, es decir, que la gestión de redes sociales en una empresa no influye de manera significativa en las utilidades, existe una baja frecuencia de publicaciones tanto en las empresas con mayores utilidades, como en las empresas de utilidades negativas consideradas en el estudio, sin embargo, el nivel de utilidades considerado para ambos listados, posee una diferencia significativa.

Palabras Clave: redes sociales; empresas; detallista; marketing; engagement

\begin{abstract}
This work aims to analyze the influence level of social networks on business success, it has been developed through two research tools, the first tool consists of an observation chart towards social networks managed by the companies selected as the object study, whose analysis was performed based on four items: activity, community, visibility and interactivity; Regarding the second tool, the use of a Likert scale survey stands out, which has made it possible to know the perception of consumers concerning the use of social networks as an information tool for their purchasing trends. The instruments applied made it possible to determine that management in social networks does not represent a key point about business success, however, of the 5 companies analyzed, less than $50 \%$ manage social networks,
\end{abstract}


allowing them to reject the hypothesis proposed, it means, that the management of social networks in a company does not affect significatively on its profits, there is a low frequency of publications in both companies, with higher profits, such as companies with negative profits considered in the study, however, the level of profits considered in both registers, has a significant difference.

Key Words: social network; business; retail; marketing; engagement

\section{Introducción}

La evolución de las redes sociales ha generado un cambio en la relación existente entre los individuos (Tricoci, Rosenthal, Corral, \& Gil, 2014). Así como el uso de las nuevas tecnologías de información ha permitido que, con su rápida evolución, aquellas disciplinas y áreas de conocimiento que tienen relación con la comunicación enfrenten un cambio radical (Cascales, Fuentes, \& Esteban, 2017). Debido a ello, en este estudio se ha tomado como un dato relevante aquellas gestiones que se pueden realizar mediante internet, tal es el caso de las redes sociales en el contexto de canal de comunicación, codificando los mensajes transmitidos empresa-consumidor, pero a su vez facilitando el flujo de información bidireccional (Martinez, 2018).

Según la encuesta multipropósito de las TIC's INEC (2018) un 37,2\% de hogares ecuatorianos poseen acceso a internet y de la totalidad de la población mayor a 5 años, un $55,9 \%$ de ecuatorianos, han utilizado un dispositivo con internet en los últimos 12 meses, de estos el 66,2\% accede desde su hogar, así como un 84,0\% de personas con acceso a internet lo utilizan al menos una vez al día. Considerando los datos expuestos, se puede obtener un precedente interesante para establecer la base de la presente investigación, puesto que se ha tomado en cuenta un considerable porcentaje de la población actual de Ecuador con acceso a internet, el uso de internet incide directamente en el impacto que tienen las empresas mediante los canales digitales dentro del país.

El impacto de la llegada y evolución del internet, ha desarrollado una necesidad en las empresas para crear su propia presencia digital, puesto que la demanda en el consumo de internet presenta un incremento como se ha establecido en los datos presentados por la encuesta multipropósito INEC (2018). Esto resulta en nuevos canales de ventas para las empresas del sector retail, así como una nueva forma de relaciones públicas y un medio para generar una comunicación bidireccional con sus consumidores, ocasionando una mayor interacción que permita conocer a los empresarios las opiniones de su target mientras estos sean gestionados de una manera correcta (Cascales, Fuentes, \& Esteban, 2017).

Para una mayor comprensión del estudio en primer lugar es necesario analizar el sector retail, considerando el segmento de empresas escogido como objeto de estudio, se puede decir que el retail hace referencia al último eslabón de la cadena comercial donde el intermediario se encarga de facilitar el acceso a los productos para un consumidor final, este detallista, tiene por principal objetivo determinar los gustos y preferencias de su target y una vez determinado, orientar sus esfuerzos a solventar esas necesidades de una forma más eficiente que sus competidores (Quintero, 2015).

Los antecedentes expuestos, permiten que el presente trabajo encuentre un campo de investigación que no ha sido explotado previamente, analizar el nivel de influencia que poseen las redes sociales ante el éxito empresarial. Se ha considerado relevante el desarrollo 
de un método que permita comprobar la relación existente entre la gestión del social media y el éxito empresarial, considerando el auge del internet en los hogares ecuatorianos y el costo de manejar contenido digital en plataformas de redes sociales a comparación del costo que implican los medios tradicionales.

Como objeto de este estudio entonces es necesario develar ¿Cuáles son los medios digitales más utilizados por las empresas?, ¿con qué frecuencia gestionan estos medios?, ¿Está su gestión bien enfocada según el público al que ostentan? Son estas interrogantes las que permitirán conocer de una forma más detallada la gestión digital de las empresas ecuatorianas y su influencia en los hábitos de compra de los consumidores.

\section{Marco Teórico}

\section{Definición de los públicos}

Las redes sociales o comunidades virtuales como mencionan Wienhausen y Andrés, (2015) poseen una lógica diferente a las comunidades físicas, considerando las distintas limitaciones que presenta la disposición de cada una de ellas, resultando el social media como una herramienta de intercambio dinámico entre personas, como grupos e instituciones, destacando que, al tratarse de comunidades virtuales sus limitaciones no son geográficas, sino están orientadas a los gustos y preferencias de los usuarios, pues estas crean grupos específicos encaminados a un mismo objetivo o a la satisfacción de una misma necesidad.

Costa, Sanches y Oliveira (2018) exponen que es necesario conocer el público objetivo al que ostenta una marca, puesto que la correcta concepción de los gustos, preferencias, hábitos de consumo, estilo de vida, emociones y motivaciones; provee la herramienta para una correcta toma de decisiones en cuanto a los esfuerzos de publicidad; tomando, así como eje fundamental los públicos que se puede encontrar en cada una de las redes sociales que se exponen a continuación como medios de estudio.

En primer lugar, se puede tomar como referencia el uso de Facebook como una plataforma de social media, considerándolo como un espacio virtual habitable (Gurevich, 2016), ya que genera presencia con los perfiles de usuarios que constantemente comparten distintos contenidos ya sea de imagen, audiovisuales o mensajería directamente; dentro de esta plataforma se puede encontrar una relación diferente al resto de redes sociales, puesto que se crea una "amistad", a diferencia de otras que persiguen el concepto de seguidores, donde no se debe aplicar necesariamente que ambos contactos se sigan mutuamente.

Facebook se concentra en la conectividad social, es por esto que puede ser tomada como una herramienta que proporcione información a los consumidores como un esfuerzo de marketing que señale datos relevantes como lanzamiento de nuevos productos, eventos y las promociones que existan en general, brindando así los recursos para que los consumidores puedan establecer interacciones referente a la marca y sus novedades, sin embargo un uso narcisista de esta plataforma, puede ser una de las limitantes para las empresas (Silva, Montero, \& Cordeiro, 2017).

Así mismo, es necesario conocer referente a Twitter, siendo esta otra de las plataformas donde existen cuentas empresariales, se puede establecer que éste es un mircoblogging nacido en 2006 que ha evolucionado las comunicaciones al ser una de las redes sociales más destacada, contando hasta el año 2014 con más de 35 idiomas de versiones 
disponibles en el mercado (Salazar \& Prieto, 2015). Los autores mencionan que es necesario que la empresa tenga en cuenta principalmente si su público objetivo se encuentra en esta red social, caso contrario sería un desperdicio de esfuerzos el gestionarla. Una vez se ha establecido que es correcta la presencia en twitter es necesario que la descripción contenga palabras clave que permitan una búsqueda fácil del perfil, así como la identificación de cuenta oficial para evitar confusiones entre los usuarios y por último la correcta gestión entre los colores de la marca y las imágenes que serán utilizadas como perfil de la marca.

Cansino (2017) expresa en primer lugar que es necesario destacar el límite de escritura dentro de esta plataforma, cuyo máximo espacio es 140 caracteres, esto radica en que los mensajes dentro de este medio deben ser cortos y concisos, además la plataforma de twitter presenta la posibilidad de postear tweetpics y tweetcams como vínculos, sin embargo, según el autor, se expone este espacio como una personalización de presencia digital de un individuo para la expresión de pensamientos, puesto que las personas están más familiarizadas con el "piar" de un "Homo Twitter", que el de una marca.

YouTube, en cambio, siendo una de las últimas redes tomadas como referencia se lo establece, acorde con Arguedas y Herrera, (2016) como una TIC que desarrolla un alto impacto, puesto que se puede acceder a ella desde una gran diversidad de dispositivos Smart, esto ayuda a que sus contenidos sean de gran relevancia entre sus usuarios debido a la accesibilidad de los mismos; esta sencillez para la plataforma, la convertiría en una de las herramientas más interesantes para las empresas al momento de gestionar su publicidad.

Pérez (2012) establece que esta herramienta es un portal de video que nació como pionero, convirtiéndose en el más visitado, obteniendo el tercer lugar entre los sitios más buscados a nivel mundial aparte del buscador Google y Facebook, este sitio web transformó el tipo de consumo de material audiovisual convirtiéndolo en bidireccional por la posibilidad de generar contenido tanto de empresas como de usuarios en general o incluso el agregar los videos cargados en esta plataforma como anexos en distintas páginas. Sin embargo, en los años más recientes se ha podido evidenciar un cambio en el servicio de esta plataforma, puesto que existe una nueva forma de percibir ganancias para los creadores de la misma, es referente a los anuncios pautados por las empresas, estos se pueden encontrar entre la reproducción de los videos cargados en YouTube.

\section{Conceptos de la Herramienta de Estudio}

El desarrollo de la herramienta de estudio se ha planteado con la finalidad de conocer en gran parte los datos de las plataformas digitales que manejan las empresas en la actualidad tomando como punto de partida el inicio del año 2019. Para esto es fundamental conocer los distintos aspectos que puede abarcar la gestión de dichas plataformas, considerando el gran impacto que poseen actualmente las redes sociales en las estrategias empresariales de marketing, tal es el caso de Facebook según lo aseguran (Sanchez, Schmidt, Zuntini, \& Obiol, 2017).

El uso de canales digitales, se ha convertido en una forma de administración para las relaciones públicas que posee una empresa, de manera digital se trabaja con transparencia, transformándose en una forma de comunicación que no solo busca dar a conocer la información sobre el producto o servicio, sino que crea ese canal bidireccional donde la base es el diálogo y la creación de una relación a largo plazo (Pérez \& Torres, 2019). Esto radica 
en la importancia de la "Actividad" que posean las marcas en sus cuentas digitales, es decir, la frecuencia de publicaciones que estas tengan por día, semana o mes.

Como lo establecen Paniagua y Gómez, (2012) las redes sociales, otorgan al usuario el poder de crear o compartir contenidos desde los perfiles que estos administren, ya sean públicos o privados, entre las redes sociales de uso más frecuente se pueden destacar, Facebook, Twitter, LinkedIn y YouTube, según los datos analizados bajo los mismo autores, entre estas existe un dato en común relevante para todas las redes previamente mencionadas, este es el número de followers o suscriptores que poseen las cuentas, dando paso así a la importancia de la "comunidad" en las redes sociales.

Cruz y Miranda, (2019) mencionan que las herramientas digitales son aquellos canales de comunicación que utilizan los clientes para obtener información, considerando el comportamiento cambiante de los consumidores actuales, estos esperan obtener la mayor cantidad de información sobre un bien o servicio, esto las vuelve un factor relevante al momento de la toma de decisión, ya que la tecnología ha marcado un hito importante en las formas de relaciones públicas de las organizaciones actuales. Los antecedentes, permiten exponer como uno de los principales factores la "Visibilidad" que posean las empresas en los medios digitales como canales de marketing en un posicionamiento SEO o SEM.

Cabe destacar entonces, ¿Qué es el SEO?, es un acrónimo que hace referencia a las siglas Search Engine Optimization, mismas que se utilizan para denominar al proceso mediante el cual una empresa obtiene y mantiene posiciones notables en los buscadores de internet de una manera orgánica o algorítmica, es decir que no es pagada, sino que el buscador en base a sus algoritmos se encarga de ordenar los resultados tomando a consideración una serie de factores (Iglesias \& Codina, 2016).

Así mismo, se puede destacar según Serrano (2016) que el $S E M$, es el contrapunto a la herramienta $S E O$, siendo su principal diferencia el costo de posicionamiento, puesto que el Search Engine Marketing (SEM), si tiene un costo por aparición o sugerencia dentro de los buscadores de internet; permitiendo que aquellos que lo contraten, obtengan reportes del alcance de sus publicaciones y menciones en la inmensidad de la web, demostrando que para un buen posicionamiento es necesaria la inversión de recursos claves como tiempo y dinero en la mantención y monitorización de contenidos generados por una marca.

En la actualidad, el uso de redes sociales, según Sánchez y Pinochet, (2017), intensifica el intercambio de información y conocimiento, ya sea esto entre amigos para los perfiles personales o, aplicado a este caso, entre la empresa y el cliente, también conocido como BtoC (Business to consumer) cuyo estudio comprende a una clasificación derivada del CRM (Customer Relationship Management), mismo que hace referencia al nivel de "Interacción" que posee el consumidor con la empresa, y esta indica escalas importantes como el nivel de enganche que tiene un consumidor, cuán importante se siente para la compañía, identificación y experiencia de compra (Demo, De Morais, Vasconcelos, \& Rozzett, 2017).

La interactividad en medios digitales, comprende un ámbito mucho más reducido de posibilidades, acorde con las características de la red que se esté empleando como medio de comunicación, esta hace alusión al contacto que poseen los consumidores con los medios digitales gestionados por la marca, por lo general caracterizados por tres indicadores que se pueden medir inclusive desde una perspectiva externa: Reacciones, Shares o Compartir y 
Comentarios ofeedback. Pues se ha establecido que los consumidores, con el objetivo de mostrar ante la sociedad aquellas experiencias de compra positivas, pues mientras mayor pueda ser demostrada aquella experiencia en medios sociales, mayor será el grado de satisfacción de consumo (Luna, 2017).

\section{Engagement, una forma de medir la actividad en redes sociales}

Acorde con Kaptein, Parvinen, y Pöyry (2015), el engagement indica una relación positiva entre la actividad de la comunidad online y la marca en sí, tomando en cuenta esta relación muchas veces como lealtad de marca, se puede decir que un incremento en la actividad online que posee una determinada marca, será favorable con el nivel de afinidad que posean los consumidores con cualquier tipo de contenido que sea gestionado a través de las redes sociales, esto permite que se cree valor ante su público o stakeholders.

El engagement ha sido un tema previamente investigado en otros campos especialmente de ciencias sociales, dentro del contexto del marketing es relativamente nuevo siendo sus principales involucraciones en herramientas como el social media, retailing y servicios, pues consiste en establecer la relación directa que posee la audiencia o público con la marca cuya medición se ha podido establecer a lo largo del tiempo ya sea de manera cualitativa o cuantitativa (Dessart, 2016).

Se puede decir entonces que el engagement, establecido por Syrek, Kühnel, Vahle y De Bloom (2018) puede referirse a diversos contextos dentro del marketing, para el objeto de estudio se tomará el concepto de redes sociales, cuya principal aplicación es la interacción mediante la tecnología y los dispositivos inteligentes incluyendo el uso de las redes sociales más utilizadas por las empresas en el contexto ecuatoriano (Facebook, Twitter y YouTube), este parámetro será medido en una forma cuantitativa en base al número de interacciones asignado previamente con un valor de importancia subjetivo versus el número de seguidores que posean las páginas como un factor de medición externa a las organizaciones evaluadas, cuyo resultado se medirá como aceptable si está sobre el $2 \%$.

\section{Metodología}

El presente estudio, ha sido realizado acorde a los parámetros que engloba una investigación cuantitativa, tomando en cuenta un análisis estadístico descriptivo dividido en dos herramientas, el primero engloba la observación, que, aun presentando un enfoque subjetivo considerando el rol del observador como lo menciona Cieza (2013) ha permitido obtener datos relevantes sobre la gestión de contenido en las redes sociales que manejan las empresas con mayores utilidades, versus las empresas con utilidades negativas del sector retail en el Ecuador.

La herramienta de observación se ha tomado en cuenta conforme a distintos conceptos previamente estudiados, dando como resultado un listado de 4 bloques que abarcan los aspectos más relevantes de una comunidad virtual: Actividad, Comunidad, Visibilidad e Interactividad; estos han sido la pauta para determinar distintos factores que pueden influir en el éxito de la gestión de medios digitales, mismos que han sido determinados con una lluvia de ideas para finalmente ser filtrados por su concordancia con los bloques finales considerados en la herramienta de estudio. 
En cuanto al último periodo, se ha establecido el levantamiento de información a través de una encuesta de escala Likert, donde se han formulado ítems que buscan determinar la importancia de la información en redes sociales para los consumidores del segmento millennial tomando en cuenta redes sociales como Facebook, Twitter, Instagram y YouTube.

Esta segunda herramienta ha sido evaluada a través de la herramienta de análisis estadístico SPSS y validada conforme los estándares del alfa de Cronbach, expresa el porcentaje de varianza permitido en una relación entre el número de ítems y el tamaño de la muestra Caycho (2017), esta última herramienta ha sido analizada mediante las medidas de tendencia central y dispersión, aplicando la media aritmética, varianza, desviación estándar y coeficiente de variación; que ha permitido evaluar las tendencias de comportamiento en los consumidores encuestados.

\section{Población y muestra}

Se ha tomado como punto de partida el listado obtenido desde la página de la Superintendencia de Compañías del Ecuador, con el reporte generado en actualización hasta el primer semestre de 2019 (mes de junio), su depuración ha dado como resultado la lista de empresas del sector retail clasificadas según su nivel de utilidades, tomando así el top 10 de las empresas con mayores utilidades y las diez empresas con utilidades negativas de esta lista, para su posterior observación y medición de engagement.

Para el cálculo de la muestra, se ha tomado como referencia la población millennial de la ciudad de Machala cuyo dato es de aproximadamente 63.706 individuos, con probabilidades de éxito y fracaso de 0.5 respectivamente, un nivel de confianza de 1,64 y un margen de error permitido de 0.06. considerando la fórmula conocida para cálculo de muestra $n=\frac{Z^{2} * P * Q * N}{E^{2}(N-1)+Z^{2} * P * Q} ;$ se ha determinado una totalidad de 114 encuestas a recolectar entre la población millennial.

\section{Planteamiento de hipótesis}

Las utilidades que percibe una empresa son uno de los aspectos más importantes en cuanto a la rentabilidad del negocio, las empresas del sector retail escogidas para su análisis han sido seleccionadas en base a su nivel de utilidades acorde con la base de datos obtenida de la página de la superintendencia de compañías con corte a Junio de 2019, es por esto que se ha planteado una relación directa entre la gestión de medios digitales con los niveles de utilidad de las empresas, esta relación da como resultado el planteamiento de la primera hipótesis.

$\mathrm{H} 1 \rightarrow$ El nivel de utilidades que presentan las empresas es directamente proporcional a la gestión que realiza en sus medios digitales, a mayor nivel de utilidades, mayor frecuencia de publicaciones.

\section{Análisis de Resultados}

De los resultados obtenidos por medio de la observación realizada a las empresas en las redes sociales que manejan actualmente, se han considerado relevantes los siguientes puntos a detallar: 
Se ha podido destacar como información general que en su mayoría las empresas gestionan sus contenidos de la siguiente manera: aproximadamente 2 de 10 publicaciones realizadas son de contenido recordatorio, dando paso a un $75 \%$ de publicaciones gestionadas con un eje persuasivo, y un 5\% restante con un contenido informativo predominante, se ha considerado en primer lugar este análisis, puesto que es necesario conocer el tipo de publicaciones que se realizan a nivel de contenido para un énfasis en la gestión y el tipo de engagement que generan este tipo de publicaciones, se puede decir que en su mayoría, las empresas buscan codificar un mensaje persuasivo que les permita incentivar el nivel de ventas.

Se toma como un dato relevante también la cantidad de publicaciones diarias, considerando que las empresas tomadas para su estudio tienen en común el tipo de sector al que pertenecen, pero a su vez, estas comercializan distintos tipos de productos. En cuanto a la frecuencia de publicación, se ha observado que una empresa tanto del top 10 de empresas con utilidades positivas, como de las 10 empresas con utilidades negativas, se destaca en un grado de publicación más frecuente, a diferencia del resto de organizaciones consideradas en el estudio, de las cuales algunas no cuentan con presencia digital en las redes analizadas (Facebook, Twitter y YouTube, descartando Instagram porque ninguna de las empresas de estudio, gestiona su contenido en esta plataforma).

A consideración de la herramienta planteada, es necesario develar en primer lugar el dato de la actividad que poseen las organizaciones en las plataformas digitales analizadas, siendo necesario destacar la frecuencia de publicaciones que poseen las primeras 4 empresas en Facebook, la empresa tomada como A1 (productos de consumo masivo), presenta una actividad de entre mínimo 6 y máximo 20 publicaciones por semana, asimismo existe para la empresa B1(Venta de Fragancias) un mínimo de 0 publicaciones por semana (considerando que en el periodo de estudio existen 6 semanas en las que no se gestionó contenido), mientras que el máximo de publicaciones semanales es de 30 en el caso de concursos y otros contenidos informativos.

La empresa $\mathrm{C} 1$ (almacén de electrodomésticos), posee una frecuencia de publicaciones semanales de mínimo 0 , existiendo un periodo de 8 semanas no consecutivas donde no se gestionó ningún tipo de contenido mientras que posee un máximo de 18 publicaciones semanales entre concursos y contenido informativo de promociones. Como último punto se puede destacar la empresa D1 (Materiales de construcción), cuya frecuencia de publicación es demasiado baja en comparación con el resto de organizaciones analizadas, considerando solo una publicación realizada en todo el periodo de estudio.

Es necesario destacar asimismo que en cuanto a la frecuencia de publicaciones en Twitter solo se ha encontrado que la empresa A1 gestionó un contenido diario durante cada semana del proceso de estudio, mientras que las empresas $\mathrm{B} 1$ y $\mathrm{C} 1$ poseen una cuenta en la plataforma, sin embargo, no cuentan con ninguna publicación con la que puedan interactuar los usuarios en el periodo de tiempo que se ha tomado como referencia de estudio.

Acorde con el segundo bloque establecido en la herramienta de estudio, es necesario detallar la información respecto a las comunidades online, se ha sostenido que dentro de la red social Facebook, la comunidad online o número de seguidores en este caso para las fan pages empresariales oscilan entre los 23.000 y 211.000 seguidores, perteneciendo la cifra menor a una empresa detallista en material de construcción (Empresa D1), mientras que el más alto, pertenece a la empresa con mayor frecuencia de gestión, cuya actividad principal es 
el comercio de productos masivos (Empresa A1), perteneciendo las dos restantes a la comercialización de perfumes (Empresa B1) y almacén de electrodomésticos (Empresa C1) respectivamente.

Tomando en cuenta, la plataforma de Twitter, únicamente se pudo encontrar que 3 de las empresas del top 10 de mayores utilidades, fomentan su presencia en dicha red social, estas son la Empresa A1 con 1.490 followers, la Empresa B1 con 1.066 y la Empresa E1 con 1.215 seguidores, tomando en cuenta el segmento de mercado al cual se dirige cada red social y cada empresa, se puede comprender el porqué de la popularidad de ellas, sin embargo, tomando en cuenta el segmento al que ostentan las empresas, es muy poco probable que encuentren su target en esta red, puesto que como se mencionó anteriormente según Cansino (2017), esta red es más utilizada para dar a conocer pensamientos y posiciones de un individuo, que la identificación de corporaciones.

Considerando, asimismo, la presencia que poseen estas empresas en la plataforma de YouTube, se ha podido observar que únicamente dos de las empresas mencionadas poseen presencia en esta red, estas son: Empresa B1 con 1.001 suscriptores y la Empresa C1 con una comunidad 3.670, sin embargo, se puede destacar que no existe una gestión periódica de las cuentas empresariales, brindándole escaso contenido a su público objetivo.

El tercer bloque destacado de análisis respecta a la visibilidad que poseen las empresas objeto de estudio considerando un nivel de posicionamiento en buscadores SEO, se puede destacar que las empresas estudiadas (esencialmente las cuatro empresas que poseen presencia en redes sociales), gestionan su contenido considerando aquellas palabras clave y hashtag que les permiten ser clasificadas de manera correcta entre los resultados de búsqueda online, ocasionando que los consumidores encuentren sus perfiles de una forma rápida.

Como último punto, se puede encontrar la interactividad, destacando que existe un nivel relativamente bajo en este punto, atribuido a las publicaciones realizadas por estas empresas, puesto que en la plataforma de Facebook, la mayor cantidad de interacciones es atribuida a la empresa A1 con un promedio de 2.299 mientras que la menor cantidad es atribuida a la Empresa $\mathrm{C} 1$ con un promedio de 120 interacciones dentro del periodo de estudio, comprados, se puede decir que el resultado de la empresa $\mathrm{C} 1$ es menos confiable puesto que su tendencia central está lejos de sus extremos, puesto que tiene un coeficiente de variación de 1,33; mientras que la empresa A1 posee un coeficiente de variación de 0,38. Este resultado demuestra que las reacciones de la comunidad ante el contenido gestionado, no son adecuadas ante el tamaño de la comunidad que se presenta.

En cuanto a la medición del engagement de la primera lista de empresas (mayores utilidades), es necesario en primer lugar, conocer los datos que se ha revelado con la observación, para esto se ha dividido la interactividad entre la comunidad, bloque 2 / bloque 4; obteniendo como resultado para la empresa A1 un porcentaje de 1,52; y para la empresa C1 0,14\%; dando paso a un análisis de engagement demasiado bajo considerando una visión externa de la comunidad en comparación a las reacciones ante la gestión de contenido. Las empresas B1 y D1 poseen valores que se encuentran dentro del rango mencionado, siendo estos 1,34 y 1,20 respectivamente (Tabla 1 ) 
Tabla. 1

Engagement de las empresas

\begin{tabular}{cccc}
\hline EMPRESA & SEGUIDORES & INTERACCIONES & ENGAGEMENT \\
\hline $\boldsymbol{A 1}$ & 211.000 & 3213 & $1,52 \%$ \\
$\boldsymbol{B} \boldsymbol{1}$ & 37.191 & 498 & $1,34 \%$ \\
$\boldsymbol{C} \boldsymbol{1}$ & 137.000 & 195 & $0,14 \%$ \\
$\boldsymbol{D} \boldsymbol{1}$ & 23.741 & 286 & $1,20 \%$ \\
\hline
\end{tabular}

Fuente: elaboración propia

En cuanto a la medición que se ha realizado para calcular el engagement de las otras dos redes sociales para cada una de las empresas, como ya se ha manifestado anteriormente, únicamente la empresa A1 ha demostrado presencia y actividad en la plataforma de Twitter, siendo así el nivel de engagement $0,58 \%$ aún muy bajo para la comunidad que poseen.

En cuanto a la plataforma de YouTube se presenta un caso similar, puesto que, de la totalidad de empresas evaluadas, dos organizaciones poseen presencia en esta plataforma, estas son la empresa A1 y la empresa C1 con un nivel de engagement de 0,17 y $0,11 \%$ respectivamente, considerándolos aún por debajo del porcentaje aceptable.

Así mismo se ha procedido a evaluar las empresas de utilidades negativas, destacando solo una entidad comercial que, con presencia en medios digitales, específicamente en las redes sociales Facebook e Instagram, dejando de lado su presencia en las demás redes sociales tomadas para el estudio que son Twitter y YouTube. La empresa tomada para el siguiente resultado se dedica a la venta de combustibles tanto para vehículos como para motocicletas en Ecuador. Constatando que solo se dedica la empresa a esta actividad.

Dando paso al siguiente dato en el cual se determinó que dentro de los comunicados realizados mantienen un perfil mayoritariamente persuasivo y con menor presencia de recordatorio, es decir, que en sus cantidades semanales tienen un $80 \%$ de perfil persuasivo, dejando el restante en el perfil recordatorio, dato que se maneja en las dos redes sociales estudiadas, es decir, 8 de 10 difusiones es la mezcla entre persuasivo e informativo. Con la intención del manejo del engagement apropiado para esta empresa con su comunidad de seguidores. Adentrando un poco más de las difusiones realizadas en dichos medios, tratan de manejar una relación entre la cantidad de compartidos y comentarios, mientras que en consideración a las reacciones que se pueden usar en estas redes sociales, esta supera de forma abismal a la relación anteriormente mencionada.

Otro punto a destacar de la observación realizada es que manejan una frecuencia de publicaciones en la red Instagram diaria, así mismo como en Facebook, tratando de incentivar a la interacción con sus seguidores ya sea por medio de las reacciones y comentarios en sus fans pages oficiales además de dar una relevancia mismo con el aspecto del SEO/SEM de esta entidad comercial.

Se tomó otro dato con respecto a la comunidad conformada por 4.625 seguidores de la cuenta oficial de Instagram, mientras que, en la cuenta oficial de Facebook, cuentan con una comunidad de 64.148 de seguidores. Del siguiente dato obtenido por la observación se identificó que dentro de sus publicaciones en la página de Instagram manejaban un promedio semanal de 8 anuncios, mientras que en la cuenta de Facebook realizaban difusiones con un promedio de 13 por semana. Además, otro punto vital de la observación misma es que 
mantenían una frecuencia de publicación casi diaria, con la intención de incentivar a su comunidad a la adquisición su servicio y así mismo a identificarse con la marca.

Otro dato destacado en la observación directa son los tipos de reacciones que se maneja en Facebook, en la cual manejan una mayor cantidad de likes. Superando por mucho a las demás reacciones que pueden usar en dicha plataforma digital. Agregando un dato relevante que dio mayor interés.

En el tercer punto hablando con respecto a la visibilidad que maneja esta empresa por medio de las redes sociales usadas para el manejo de su marca, ha optado por un manejo SEO, la cual desarrolla por medio del hashtag y las palabras clave que mantienen una sintonía que expresa la marca en sus difusiones y a la vez que ésta sea encontrada rápidamente por los consumidores en los perfiles sociales.

Y con el último asunto la cual es la interactividad que posee la marca con su comunidad digital, tomando a la red social Facebook maneja una mayor cantidad de interacciones como es el punto de las reacciones contando con promedio más alto de 2600 aproximadamente y uno más bajo 1000, mientras con respecto a los comentarios que mantiene como punto más bajo un aproximado de 50 y como punto más alto está oscilando entre los 800 y 900, mientras que en la red social Instagram esta mantiene una cantidad de reacciones con un aproximado que oscila entre 1 y 10 como punto más bajo y su mayor cantidad que está entre los 100 y 120 , y así mismo con respecto a los comentarios que mantiene cada difusión entre 15 y 20 como punto más alto y su punto más bajo que oscila entre 1 y 5 en sus publicaciones del periodo de muestra tomado.

Para concluir con el punto del engagement, se ha tomado en primer lugar Facebook cuyo resultado arroja un 5,3\% mientras que en el análisis realizado a la red social Instagram presentó un engagement de 1,08\% el cual se encuentra por debajo del porcentaje aceptable, cabe recordar que la comunidad digital que tiene por esta red es totalmente baja en comparación a Facebook, por dicho motivo se expresa que la marca por este medio no se apega mucho a lo que sus seguidores quieren.

En cuanto al levantamiento de información a través de la encuesta de escala Likert, cuya evaluación se ha realizado a través del cálculo de la media, Varianza, Desviación Estándar y Coeficiente de Variación, se ha podido obtener los siguientes resultados: expresando la primera variable de evaluación se ha descubierto una media de 2,75; y una desviación estándar de 1,17 donde se hace referencia a la importancia del internet en la decisión de compra de los consumidores, para la siguiente variable que menciona cuán importante es para los individuos seguir las marcas de los productos y servicios que consumen en redes sociales, presenta una desviación estándar de 1,40 con una media de 3.24; otorgándole un rango de dispersión bastante amplio dentro de la escala escogida.

La red social Facebook posee dos variables, la primera refiere la confianza de los usuarios ante la información que encuentra en esta plataforma, se ha obtenido una media de 2,86 y una desviación estándar de 1,3 mientras que la segunda variable se plantea la bú squeda de la marca en este medio digital, ante un nivel de desconocimiento presentando una media de 3,61 y así mismo una desviación estándar de 1,28. Posteriormente se ha evaluado el nivel de uso que los usuarios otorgan a los comentarios para expresar un desacuerdo con las marcas demostrando una media de 2,64 y una desviación estándar de 1,55. 
Como último punto con respecto a la herramienta utilizada, se ha evaluado el uso de Instagram, Twitter y YouTube como herramienta de búsqueda, donde los usuarios encuestados expresaron a Instagram como fuente de información en una media del 3,08 con una desviación estándar de 1,42; mientras que Twitter demostró una media de 1,66 con una desviación estándar de 0,96; concluyendo con YouTube el cual presentó una media de 2,49 y una desviación estándar de 1,47 en cuanto a su utilidad como herramienta de búsqueda y una desviación estándar de 1,47 en cuanto a su utilidad como herramienta de búsqueda.

Tabla. 2

Uso de Instagram, Twitter y YouTube

\begin{tabular}{lcccc}
\hline \multicolumn{1}{c}{ VARIABLELMEDIDA } & MEDIA & $\begin{array}{c}\text { DESV. } \\
\text { EST. }\end{array}$ & VARIANZA & $\begin{array}{c}\text { COEF. } \\
\text { VAR }\end{array}$ \\
\hline Baso mis decisiones de compra en internet & 2,75 & 1,17 & 1,37 & 0,43 \\
Sigo las Marcas & 3,24 & 1,40 & 1,95 & 0,43 \\
Confí en la información de Facebook & 2,86 & 1,30 & 1,68 & 0,45 \\
$\begin{array}{l}\text { Busco una página de Facebook cuando no conozco la } \\
\text { marca }\end{array}$ & 3,61 & 1,28 & 1,64 & 0,35 \\
Uso comentarios para expresar un desacuerdo & 2,64 & 1,55 & 2,41 & 0,59 \\
Busco información en Instagram & 3,08 & 1,42 & 2,02 & 0,46 \\
Busco información en Twitter & 1,66 & 0,96 & 0,92 & 0,58 \\
Busco información en YouTube & 2,49 & 1,47 & 2,16 & 0,59 \\
\hline
\end{tabular}

Fuente: elaboración propia

\section{Conclusiones}

En primer lugar es necesario destacar que el $40 \%$ de las empresas con mayores utilidades así como un $10 \%$ de las empresas con utilidades negativas demuestra presencia en redes sociales, por lo tanto no se considera a los medios digitales como un factor de éxito empresarial, puesto que no afecta al nivel de ingresos que han obtenido las organizaciones, únicamente se podría destacar la gestión de estas herramientas como un apoyo para la comunicación B2C, mas no como un factor determinante para incrementar su nivel de utilidades.

De la totalidad de empresas evaluadas se ha observado amplias comunidades digitales con una gestión de contenido frecuente y en algunos casos poco frecuente, además, de constatar que existen empresas que no gestionan contenido online, esto permite contrarrestar la hipótesis plateada, ya que se consideró que la gestión de contenido y las utilidades de una empresa NO poseen una relación directamente proporcional, como se había planteado en primer lugar, destacando que un bajo porcentaje de empresas demuestran presencia en redes sociales, siendo Facebook la más exitosa, seguida por YouTube y en último lugar Twitter e Instagram debido al tipo de público que ostentan concordando la posición de Facebook y Twitter a comparación con la encuesta donde los consumidores evaluaron el nivel de importancia para su búsqueda de información en redes sociales, sin embargo, Instagram, aun demostrando mayor relevancia en la percepción de los consumidores presenta una escasa gestión por parte de las empresas, se puede inferir que esta situación es ocasionada por el público objetivo de la herramienta versus el público de la empresa.

Considerando el tipo de sector analizado, enfocado en los bienes, se puede descubrir un resultado estrechamente relacionado en el contexto argentino como lo exponen Tricoci, 
Rosenthal, Corral y Gil (2014), donde las empresas de servicios resaltan su presencia en redes sociales, a diferencia de las que ofertan bienes (como lo es el sector retail), así como a comparación del análisis realizado por Moscato y Moscato (2011). En su trabajo se concluyó que, así como existen empresas que poseen presencia en redes sociales, hay otras que posiblemente por su cartera de productos, no lo consideraban relevante, sin embargo, esto no disminuye la posibilidad que se encuentre la información necesaria sobre los perfiles de los consumidores gracias a herramientas como las redes sociales.

\section{Bibliografía}

Arguedas, C., \& Herrera, E. (2016). Implementación de un canal en YouTube para apoyar un curso Física. Atenas, II(34), 1-9. Obtenido de https://www.redalyc.org/jatsRepo/4780/478054643005/478054643005.pdf

Cansino, C. (Septiembre- Diciembre de 2017). Viejas y Nuevas Tesis sobre el Homo Twitter. Revista Mexicana de Ciencias Políticas y Sociales, LXII(231), 389-405. Obtenido de https://www.redalyc.org/articulo.oa?id=42152785015

Cascales, G., Fuentes, L., \& Esteban, J. (2017). La interacción de los Hoteles con las Redes Sociales: Un Análisis de los Hoteles de Cuatro Estrellas de la Comunidad de Madrid (España). Cuadernos de Turismo, 131-148. doi:http://dx.doi.org/10.6018/turismo.39.290471

Caycho, T. (2017). Intervalos de confianza para el coeficiente Alfa de Cronbach: aportes a la investigación pediátrica. Acta Pediátrica de México, XXXVIII(4), 291-292. doi: 10.18233/APM38No4pp291-2941440

Cieza, F. (2013). La observación entre lo objetivo y lo subjetivo. Cuadernos de la Facultad de Humanidades y Ciencias Sociales(44), 23-40. Obtenido de https://www.redalyc.org/articulo.oa?id=18532860002

Costa, S., Sanches, J., \& Oliveira, F. (Julio-Diciembre de 2018). Trends Studies' Contributions For The Definition of the Fashion Brand's Target. ModaPalavra, 11(22), 268-292. doi:https://doi.org/10.5965/1982615x11222018240

Cruz, I., \& Miranda, A. M. (Abril-Junio de 2019). La adopción de las tic en restaurantes de Puerto Nuevo, Rosarito, Baja California*. Innovar, 29(72), 59-75. doi:10.15446/innovar.v29n72.77932

Demo, G., De Morais, E., Vasconcelos, D., \& Rozzett, K. (Mayo-Junio de 2017). Customer Relationship Management scale forr B2C Market: A Cross-Cultural Comparison. RAM. Revista de Administração Mackenzie, 18(3), 42-69. doi:http://dx.doi.org/10.1590/1678-69712017/administracao.v18n3p42-69

Dessart, L. (2016). Capturing consumer engagement: duality, dimensionality and measurement. Journal of Marketing Management., XXXII(5-6), 399-426. doi:10.1080/0267257X.2015.1130738

Gurevich, A. (2016). El Tiempo Todo en Facebook. Aposta. Revista de Ciencias Sociales(69), 217-238. Obtenido de https://www.redalyc.org/pdf/4959/495952431008.pdf

Iglesias, M., \& Codina, L. (2016). Los cibermedios y la importancia del posicionamiento de buscadores (SEO). Opción, 32(9), 929-944. Obtenido de https://www.redalyc.org/pdf/310/31048482052.pdf

INEC. (2018). Tecnologías de la información y Comunicación - Encuesta Multipropósito TIC 2018. INEC. Obtenido de https://www.ecuadorencifras.gob.ec/documentos/webinec/Estadisticas_Sociales/TIC/2018/201812_Principales_resultados_TIC_Multipropo sito.pdf 
Kaptein, M., Parvinen, P., \& Pöyry, E. (2015). The Danger of Engagement: Behavioral Observations of Online Community Activity and Service Spending in the Online Gaming Context. International Journal of Electronic Commerce., 20(1), 50-75. doi:10.1080/10864415.2016.1061414

Luna, G. (Abril- Junio de 2017). La influencia del consumo simbólico en la intensidad de uso de las redes sociales digitales y el valor percibido de las experiencias. Innovar, 27(64), 37-50. Obtenido de https://www.redalyc.org/pdf/818/81850404004.pdf

Martinez, C. (Enero-Junio de 2018). Uso de redes sociales en las revistas científicas de la Universidad de Los Andes, Venezuela. E-Ciencias de la Información, VIII(1), 32-52. doi: $10.15517 /$ eci.v8i1.28104

Moscato, D., \& Moscato, E. (2011). An analysis of how companies in diverse industries use Social Media in E-Commerce. International Journal of the Academic Business World, $V(2), 35-42$. Obtenido de http://web.b.ebscohost.com/ehost/detail/detail?vid=0\&sid=7260a7d8-fc12-40a3-8f10c6232a602458\%40pdc-vsessmgr01\&bdata=Jmxhbmc9ZXMmc210ZT1laG9zdC1saXZl\#db=buh\&AN=828558 02

Paniagua, F., \& Gómez, B. (2012). HACIA LA COMUNICACIÓN 2.0. El uso de las redes sociales por parte de las universidades españolas. ICONO 14. Revista de comunicación y tecnologías emergentes, 10(3), 346-364. doi:10.7195/ri14.v10i3.473

Pérez, A., \& Torres, R. (Abril-Junio de 2019). Las agencias de empleo y desarrollo local, y el uso de las redes sociales en la promoción turística relacional. Innovar, 29(72), 77-88. doi:DOI: 10.15446/innovar.v29n72.77933

Pérez, J. (2012). La actualidad en YouTube claves de los videos más vistos durante un mes. Global Media Journal, IX(17), 44-62. Obtenido de https://www.redalyc.org/articulo.oa?id=68723565004

Quintero, L. (2015). El sector retail, los puntos de venta y el comportamiento de compra de los consumidoresde la base de la pirámide en la comuna 10 de la ciudad de Medellín. Revista Ciencias Estratégicas, 23(33), 109-118. Obtenido de https://www.redalyc.org/pdf/1513/151345259009.pdf

Salazar, S., \& Prieto, P. (2015). Gestión y Administración de la comunicación institucional en Twitter. Anuario Electrónico de Estudios en Comunicación Social "Disertaciones", VIII(1), 1-18. Obtenido de https://www.redalyc.org/jatsRepo/5115/511553138003/511553138003.pdf

Sánchez, M., \& Pinochet, G. (Enero-Junio de 2017). El rol de las redes sociales virtuales en la difusión de información y conocimiento: estudio de casos. Universidad \& Empresa, 19(32), 107-135. Obtenido de https://www.redalyc.org/articulo.oa?id=187247578006

Sanchez, M., Schmidt, M., Zuntini, J., \& Obiol, L. (2017). La influencia de las redes sociales virtuales en la difusión de información y conocimiento: estudio de PYMES. Revista Iberoamericana de Estratégia, 16(4), 69-90. doi:10.5585/riae.v16i4.2522

Serrano, J. (2016). Crítica del Libro "SEO: Introducción a la Disciplina del posicionamiento en buscadores". Revista española de Documentación Científica, 39(3), SD. Obtenido de http://redc.revistas.csic.es/index.php/redc/article/view/952/1421

Shaw, L. (12 de Agosto de 2017). Bloomberg. Recuperado el 1 de Enero de 2020, de Bloomberg: http://web.a.ebscohost.com/ehost/detail/detail?vid=4\&sid=4b77c635f51d-41b2-b4404747701f5c1c\%40sessionmgr4006\&bdata=Jmxhbmc9ZXMmc210ZT1laG9zdC1saX $\mathrm{Zl} \# \mathrm{AN}=140648908 \& \mathrm{db}=\mathrm{buh}$

Silva, N., Montero, E., \& Cordeiro, M. (Julio-Septiembre de 2017). Facebook "Verde Amarelo" e preferencia de uso da rede para os Brasileiros. Revista de Administração

Esta obra se comparte bajo la licencia Creative Common Atribución-No Comercial 4.0 International (CC BY-NC 4.0) 
da Universidade Federal de Santa Maria, 10(3), 452-473. doi: 10.5902/19834659 12278

Syrek, C., Kühnel, J., Vahle, T., \& De Bloom, J. (2018). Ecological momentary assessment to examine the relationship between non-work social media use at work and work engagement. Work \& Stress., XXXII(3), 209-227. doi:10.1080/02678373.2017.1367736

Tricoci, G., Rosenthal, A., Corral, P., \& Gil, P. (2014). Una mirada sobre el impacto del uso de las redes sociales en las empresas argentinas. Forum Empresarial, II(1), 1-7. Obtenido de https://www.redalyc.org/articulo.oa?id=63160182001

Wienhausen, M., \& Andrés, M. (2015). Facebook en Jujuy. Uso de las redes sociales. Una discusión entre lo público y lo privado. Cuadernos de la Facultad de Humanidades y Ciencias Sociales- Universidad Nacional de Jujuy(47), 11-39. Obtenido de https://www.redalyc.org/articulo.oa?id=18547708001 